\title{
A Review of Bilateral Teleoperation Control Strategies with Soft Environment
}

\author{
Yaru Deng, Yushan Tang, Bo Yang, Member, IEEE, Wenfeng Zheng, Member, IEEE, Shan Liu, \\ Chao Liu, Senior Member, IEEE
}

\begin{abstract}
In the past two decades, bilateral teleoperation with haptic feedback has attracted great research and application interests in both robotics and other areas. Initially triggered by the need to handle dangerous and remote distance tasks such as nuclear materials manipulation and space exploration, bilateral teleoperation has found its way into other applications as a result of development of control theory, robotic technology (both hardware and software) and latest breakthrough in artificial intelligence and machine learning. Consequently, bilateral teleoperation is found facing new challenges brought by these new applications. One major and obvious change is the working environment for the slave manipulator: different from rigid or solid contact environments which are reasonably assumed in early applications in industrial, nuclear and aerospace applications, the slave environment is now more complex and often the objects in contact are much softer in term of stiffness and can not be described by simple elastic model if good teleoperation performance (accurate and transparent) is expected. In this paper, the research of bilateral teleoperation system considering soft environment in recent 20 years has been surveyed for the first time in literature, to the knowledge of the authors. Following the difference in real applications, in this review the definition of soft environment covers linear elastic environment with much lower stiffness than conventional industrial environment and nonlinear complex soft environment with/out time-varying characteristics. Accordingly, the surveyed control strategies and structures in recent literature to improve the stability and accuracy of bilateral teleoperation with soft environment are classified and explained. Finally, the main applications, current challenges and future perspectives of bilateral teleoperation with soft environment are discussed.
\end{abstract}

Keywords: Bilateral teleoperation, soft environment, passivity, transparency

\section{INTRODUCTION}

Teleoperation refers to the operation at a distance, which means that the operator of the master provides the environmental conditions that are communicated with the location of the slave to enable it to have the ability to control the object remotely. The first unilateral teleoperation allows humans to manipulate the master manipulator and provide motion instructions to the slave manipulator through a communication channel, so as to control the slave manipulator to perform operations. Unilateral teleoperation has been widely used in daily life, such as remote grasping,

This work was jointly supported by the Sichuan Science and Technology Program (2021 YFQ0003, 2021 YFS0015) and the Fundamental Research Funds for the Central Universities (ZYGX2019J059).

Yaru Deng, Yushan Tang, Bo Yang*, Wenfeng Zheng and Shan Liu are with the School of Automation, University of Electronic Science and Technology of China, Chengdu 610054, Sichuan, P. R. China. (*boyang@uestc.edu.cn); Chao Liu is with the Department of Robotics, LIRMM, University of Montpellier-CNRS, Montpellier, France. dangerous environment operation and other fields, Unilateral teleoperation enables human to manipulate the remote slave manipulator from a certain distance and the control method is simple. However, due to the lack of signal feedback from the slave site to the master device, the operator on the master side cannot intuitively feel the environment interacting with the slave manipulator, so it cannot control the slave object accurately especially when interaction force is considered. With the increasing demand for remote operation precision and safety, the teleoperation system has been expected to perceive the environment which motivates the development of bilateral teleoperation system.

In the 1940s, Goertz [1] built the first mechanically controlled bilateral teleoperation robot, which is considered to be the beginning of the development of force feedback bilateral teleoperation. In 1954, he used electrically driven position-feedback automatic controls to separate the robot's master and slave arms, now commonly named as master and slave. In the early 1960s, the communication delay in bilateral teleoperation system was taken into consideration and studied by researchers, and a series of methods (such as supervisory control [2] and predictive control [3]) were proposed to reduce its negative effects on the force feedback bilateral teleoperation system. Later on, more advanced control methods have been proposed, such as Lyapunov analysis method [4], internal virtual model [5], scattering theory [6] passive control theory [7] and wave variable method [8] etc. These theories have all contributed to the improvement of bilateral teleoperation system stability.

It is noticed that the earliest bilateral teleoperation works were mostly dedicated to industrial production scenarios, where the slave environment generally involves rigid environment, such as grasping hard objects and manipulating rigid pieces, hence most existing bilateral teleoperation results are based on such explicit or implicit assumption of the slave environment being pure elastic and interaction deformation is small. However, as a matter of fact, with the recent development of technology and social needs, the application of bilateral teleoperation robots in daily life is also gradually expending, and their interactions with the environment is more and more complicated. Bilateral teleoperation systems used in medical, service and collaborative robots all involve non-rigid and not pure elastic environments, so the existing research based on rigid environment may no longer be effective or feasible to provide satisfactory performance. In such situation, the bilateral teleoperation scheme may need re-examination.

Different from conventional rigid environment which generally possesses elastic model with constant and high stiffness parameter, soft environments show different and often much more complicated properties. In view of whether 
the soft environment is pure elastic and whether the mechanical properties change linearly, this paper divides the slave environment into simple soft environment and complex soft environment when surveying papers in the past 20 years. Simple soft environment generally refers to the environment with linear elastic model but small stiffness coefficient, whose environmental characteristics rarely change along time but deformation is easily observed and measurable. The complex soft environment generally refers to the non-elastic and nonlinear environment with/out time-varying characteristics, which can not be described by simple spring model. The most common are soft objects constituted of uneven materials or various tissues of the human body. This kind of complex soft environment brings great challenges to the design of bilateral teleoperation system.

In designing a bilateral teleoperation system applied to abovementioned soft environment, it is necessary to consider the complicated problems such as physiological fremitus of human organs, flow pressure of blood vessels, which has higher requirements for the robustness and stability of bilateral teleoperation system. Moreover, when bilateral teleoperation is carried out in complex soft environments such as human body, the real-time performance of the system is particularly important, because the characteristics of the environmental are time-varying. If the communication delay is large, the change of the contact force from the slave can not be detected in time, which will lead to the disconnection of the perception between the master and the slave. In bilateral teleoperation with force feedback, time correlation and precision of force feedback are the main indicators to measure the transparency of bilateral teleoperation. Especially in the complex soft environment, the force feedback received by the master and the environmental force received by the slave need to achieve zero time delay and zero error as far as possible, so as to provide operator with a sense of immersive experience. This makes the design of bilateral teleoperation system based on complex soft environment more challenging than the design of general rigid or simple soft environment.

In this paper, recent research works on bilateral teleoperation systems with simple and complex soft environments in the past two decades have been surveyed, and they are classified and described according to different control strategies and different specific environments (simple soft environment and complex soft environment). This paper explores their respective advantages and disadvantages in improving the design of bilateral teleoperation system based on soft environment, which provides a systematic overview of the relevant works reported in recent literature. To the authors' knowledge, this paper presents the first review on this growing topic. The end of this paper describes the existing applications of bilateral teleoperation with complex soft environment, summarizes current difficulties and challenges encountered in the design of bilateral teleoperation system for complex soft environment, and indicates potential future research hotspots and development directions.

\section{Bilateral TElEOPERATION CONTROL STRATEGY BASED ON SOFT ENVIRONMENT}

Since the concept of bilateral teleoperation was first proposed by Goertz [1] in 1949, bilateral teleoperation has developed for more than 70 years. During the 50 years of the 20th century, bilateral teleoperation system has developed in an accelerated way. The main research achievements were concentrated in the 1980 s and 1990s at the end of the 20th century. In the 1960s, research on bilateral teleoperation system mainly focused on industrial applications, such as aerospace [9] and industrial robots [10]. In the 1970s, researchers not only extended the application of bilateral teleoperation to submarine operations [11], but also studied how to optimize force feedback, considering the performance of force feedback controllers in the control system [12], adding force sensors [13], etc., and starting to study the time-delay problem in bilateral teleoperation system [14]. In the 1980s and 1990s, researchers proposed a variety of control strategies to optimize the experience of bilateral teleoperation systems. Anderson et al. [15] proposed a control strategy based on passivity and scattering theory to solve the time delay problem. Niemeyer et al. [8] used a physically motivated, passivity-based formalism to provide energy conservation and stability guarantees in the case of transmission delays, and the notion of wave variable is utilized to characterize time-delay systems and leads to a new configuration for force-reflecting teleoperation. The experimental results show the effectiveness of wave variable method. Bejczy [16] used the phantom robot predictive display to deal with the time delay problem of real robot motion. In order to improve the transparency of the system, Tesar et al. [17] proposed a force-reflecting, a 3 degree of freedom (DOF) spherical manual controller. At the same time, an impedance controlled force reflecting hand controller [18] is also used to improve the transparency of the system. Adaptive control strategy [19] and neural network predictive control [20] are also used in bilateral teleoperation system at this time. During this period, there were also some researches on control structures, such as four-channel control structure [21] and two-channel control structure [22]. These research results laid a foundation for the formation of our knowledge system in recent years.

Up to now, the control strategies of bilateral teleoperation system have been very rich, and many can be applied to soft environment, such as adaptive control, predictive control, sliding mode control, etc. There are also some hybrid control strategies combining the advantages of various control strategies, and optimization of control structure, etc. A big part of them is aimed at the simple soft environment, and other control strategies are aimed at the complex soft environment. In the following, these results are categorized and introduced according to the different environments applied to and control techniques used.

\section{A. Simple Soft Environment}

\section{1) Adaptive control}

Adaptive control strategy is widely used in bilateral teleoperation systems because it enables the system to modify its own characteristics to adapt to the change of the dynamic characteristics of objects and disturbances. For example, in paper [23], the model reference adaptive control scheme was applied to the new force control system, and in order to satisfy the requirements of rise time, stabilization time and overshoot from simulations, a third-order relative single reference model was selected. An adaptive feedback control scheme was designed to provide stability, and an adaptive feedforward control scheme was designed to compensate the disturbing force from the haptic device. The proposed control 
strategy shows good force tracking performance and stability in a simple soft environment modeled as a spring-damping model. In 2020, Bao et al. [24] proposed an adaptive finite time synchronization control scheme based on radial basis function network (RBFN), which uses RBF network to transform the system uncertainty into a series of linear combination Gaussian functions. Then, by splitting the input dead zone into a continuous function and a bounded disturbance, it is resolved. An asymptotically stable compensator and finite-time convergent updating rates are designed to achieve adaptability of the closed-loop system. Finally, the effectiveness of the proposed method is verified through simulation and actual experiment. In the actual experiment, the environment is an empty plastic bottle, which can be regarded as a simple soft environment.

\section{2) Fuzzy control}

In bilateral teleoperation system, fuzzy logic controller can be used to solve nonlinear dynamics and related uncertainty problems. For example, in order to improve the stability and transparency in telesurgery, aiming at the nonlinear and uncertain dynamics problems in bilateral teleoperation system, Kolbari et al. [25] proposed an interval type-2 fuzzy logic controller in 2016, which can control the position of the system without knowing the dynamics of the master-slave robot. It has a good control effect on the uncertainty caused by changing system parameters and sensing noise in teleoperation setting. However, this controller has high damping and slow response speed, and the time delay of bilateral teleoperation system is not considered. In 2019, Lu et al. [26] proposed a hybrid force/position control method based on fuzzy observer. Based on Takagi-Sugeno fuzzy inference rules, a position observer was designed to estimate the remote delay state at low sampling rate. Finally, it is verified that this method has the best motion synchronization effect in soft environment.

\section{3) Sliding-mode control (SMC)}

Sliding mode control is also used in bilateral teleoperation system. For example, in 2010, Hace et al [27] studied the SMC design method for bilateral control of master-slave teleoperation robot architecture based on haptic, proposed a chattering - free sliding mode control design method. In addition, the effectiveness of the proposed control strategy is verified by comparative experiments in hard environment and simple soft environment (sponge). In 2018, Salinas et al. [28] proposed a control scheme based on the combination of sliding mode control (SMC) and PD-like structure for mobile robot bilateral teleoperation system to deal with asymmetric and time-varying delays. This scheme adopts both $\mathrm{P}+\mathrm{d}$ control and SMC control strategy at the slave end, which makes full use of the advantages of the two methods, and simulation results on a simple soft environment which modeled as a damp-like model show that the proposed control strategy has good robustness and stability.

4) Model predictive control (MPC)

Predictive control is also common in bilateral teleoperation. For example, Slama et al. [29] proposed a bilateral predictive controller for a scalable teleoperation system with variable transmission delay, slave force feedback and packet loss. This method allows us to consider the slave force feedback of changing the reference trajectory in the model predictive control. In this paper, sponge and other materials belonging to simple soft environment are used in the slave. The experimental results show that the scheme is robust even though the communication delay and packet loss are variable.

\section{5) Hybrid control strategy}

After years of research, in order to improve the stability and transparency of bilateral teleoperation, researchers often take the advantages of these control strategies and combine them to form a variety of hybrid control strategies. For example, in 2020, Sun et al. [30] designed two control methods in the teleoperation system based on nuclear magnetic resonance to improve the long-distance motion and fine motion of the robot, and adopted the nonsingular fast terminal sliding mode (NTFSM) in the controller design. In order to improve the maneuverability of the system, a series of fuzzy algorithms are proposed to adjust the attitude, position, speed and force of the robot. The experiment of picking up and moving plastic bottles by robot proves the feasibility of the control method in simple soft environment.

6) Innovation of control structure

In addition to these common control strategy innovations, in order to consider the stability and transparency of bilateral teleoperation system, researchers also made a series of innovations in control structure design. There are four-channel structure and two-channel structure, and a three-channel control structure. In 2013, Suzuki et al. [31] proposed a frequency domain damping (FDD) design method for a four channel bilateral acceleration control system with communication disturbance observer (CDOB) based on modal space analysis. The experiment and analysis results in hard contact (aluminum) and soft environment (sponge) show that the method improves the stability of contact motion and environment and maintains good operability. Rossi et al. [32] also proposed a bilateral teleoperation architecture based on adaptive buffer and Smith predictor in 2017. Because prediction and adaptation make the system less sensitive to time-varying delay, packet loss and measurement noise, the system can work well in simple soft environment like spring and free motion. The teleoperation system has high precision in the setting of single degree of freedom.

\section{B. Complex Soft Environment}

Most of the control strategies for complex soft environment are driven by medical demand. In the next of this paper some control strategies based on complex soft environment are introduced. In the research of control strategy for complex soft environment, one of the early works is the design of a remote-control controller for haptic detection and soft environment researched by Cavusoglu et al. [33]in 2002. In the framework of Task-based optimization, the design problem of bilateral teleoperation controller is expressed as the optimization of a new teleoperation fidelity metric, and the constraints of free space tracking and robust stability of the system under uncertainty of environment and manual operation are considered. The experiments in [33] took the uncertainty of environment into account and the proposed new fidelity measurement method is also very suitable for telesurgery.

Since the use of nonlinear force/position mapping in soft tissue is very good at shaping the surgeon's perception, but the design of such nonlinear mapping requires a very 
comprehensive study of human factors, which is difficult to achieve. So Malysz et al. [34] focus on the design of teleoperation controllers which can reinforce expected position scaling and monotonic nonlinear force, they proposed an adaptive master-slave teleoperation controller which can handle nonlinear position/force mapping, and the expected monotone nonlinear mapping is established between the master and slave in the presence of dynamic uncertainties. In 2009, they improved the controller in [34]. Under the condition of uncertainty of dynamic parameters, the new controller can establish the filtering mapping relationship and the expected monotone nonlinear scale between the master-slave. In the case of force and position linear filter mapping, the exact fully stable region is determined [35].

In 2011, based on the application of robot assisted puncture needle in the minimally invasive percutaneous surgery, in order to realize the accurate tracking of the needle in the free motion and during insertion, Dehghan et al. [36] adopted an impedance controller at the master of bilateral teleoperation system and an impedance controller based on sliding mode at the slave to compensate for the uncertainty of the slave. In 2014, Li et al. [37] proposed a passive controller based on adaptive model. By combining the on-line estimation of human tissue model with the passive observer at the slave, the system can be improved to keep the system passive while achieving high transparency. In 2017, Li et al. [38] proposed a double closed-loop control algorithm based on current and force closed-loop when designing a needle insertion teleoperated control system based on haptic feedback, which realized the accurate matching between the feedback force and the force signal of the slave. Experiments show that the method is suitable for all haptic interfaces with force sense, but the problem of time delay has not been solved here, and the response time is long.

In 2019, in order to verify the applicability of the proposed fidelity evaluation index of virtual surgery force feedback in virtual thoracoscopic surgery, Hu et al. [39] used a sliding mode control system based on DOB(disturbance observer) to eliminate the influence of gravity, friction, parameter changes and slip of the linear drive, and improved the transparency of the force interaction series mechanism. The virtual video-assisted thoracoscopic surgery environment is modeled as a complex soft environment where the force and stiffness will change. Minelli et al. [40] proposed a new multi-master-multi-slave two-layer architecture based on the concept of shared energy tank to ensure the stable interaction between bilateral teleoperation system and slave environment in remote surgery. This structure allows to minimize the conservatism due to passivity preservation and increase transparency.

In 2020, a control method for bilateral teleoperation of 7-DOF robot with Remote Center of Motion (RCM) constraint was proposed by $\mathrm{Su}$ et al. [41]. The controller combined the decoupling Cartesian admittance control and the strategy with pedal switch based on two-layer method to realize RCM constraint. However, this paper only conducts experiments on the platform built in the laboratory to verify the stability and transparency of the system. The environment side is only an organ model, not a real human organ. Guo et al. [42] proposed a fuzzy PID controller of force feedback control system in order to improve the accuracy and response speed of master-slave vascular interventional surgery robot with force feedback, and verified its performance through master-slave force feedback experiment. Experimental results show that the feedback accuracy and the response speed of the fuzzy PID controller are better than traditional single PID controller. However, the experiment in this paper was only carried out on the model of isolated blood vessels, and no real animal experiment was carried out.

\section{APPLICATIONS AND CHALLENGES}

\section{A. Applications}

At present, the applications of bilateral teleoperation system with soft environment are mostly found in medical treatment and rehabilitation, service robots, as well as cooperative robots that can accomplish tasks together with human beings.

The applications of bilateral teleoperation system in medical field can be roughly divided into soft tissue palpation and stiffness recognition, heart surgery, vascular intervention surgery, robot assisted needle insertion, etc. The main purpose of soft tissue palpation is to identify the stiffness of soft tissue. For example, based on the stiffness characteristics of tumor, Talasaz et al. [43] applied bilateral teleoperation system to minimally invasive tumor localization surgery in 2010. It is feasible to combine haptic feedback and force feedback to improve the overall performance of tumor localization. The experimental results also prove that this method can not only detect tumor through force reflection but also improve the accuracy of tumor detection. In 2018, Aoki et al. [44] applied bilateral teleoperation system to haptic forceps in brain surgery. The use of bilateral teleoperation system enables the master and slave to transfer haptic to each other, and scale the information received from each other, which can correctly measure the environmental stiffness. Bilateral teleoperation system is often used in robot assisted cardiac surgery. For example, Liu et al. [45] studied the force control method of robot assisted cardiac surgery in 2011, proposed a three-dimensional force control method considering viscoelastic interaction, and demonstrated the robustness of the control method to measurement disturbance and model uncertainty through experiments and simulation.

Bilateral teleoperation system is also very important in minimally invasive vascular interventional surgery. In 2018, Bao et al. [46]innovated the force feedback estimation method in minimally invasive vascular interventional surgery, and proposed a novel method comprising compensatory force measurement and multimodal force feedback (MFF). This method can accurately measure the proximal force of the catheter and make it easier for the surgeon to distinguish proximal force changes. In the same year, Guo et al. [47] studied the haptic feedback in the robot assisted catheterization system, develop a real-time master slave interventional surgical robotic system with a closed-loop force feedback, which provide adequate haptic feedback to surgeon, and improve the control accuracy. In 2020, Guo et al. [42] proposed a fuzzy PID controller for force feedback in minimally invasive vascular interventional surgery, which can improve the accuracy and stability of master-slave force feedback.

In robot assisted needle insertion system, many researchers have also made innovations, mainly focusing on 
force feedback and haptic feedback. In 2011, Dehghan et al. [36] designed a hybrid control strategy to realize the accurate tracking of the needle in the free motion and during insertion in the robot assisted needle insertion system, which realized the appropriate force tracking and ensured the stability of the system in the uncertain environment. In 2017, Shin et al. [48] proposed a master-slave robot system for needle indentation and insertion, which has the ability to describe the interaction with soft tissue.

Bilateral teleoperation system can also be used in many remote services. Atashsar et al. [49] proposed a bilateral teleoperation design framework independent of passivity for network telerehabilitation system. They proposed a stable scheme for the network telerehabilitation system, which can ensure the stability no matter what the therapist's specific action is. In 2015, Tufail [50] developed a complete teleoperation system capable of effective real-time execution, and focused on the application of bilateral teleoperation system in remote homecare robotics, which are used to care care for the elderly, children and the disabled, etc. In 2017, Atashsar et al. [51] designed a new bilateral rehabilitation teleoperation robot structure, which incorporated the supervision of local/remote human physiotherapists into the rehabilitation system, and allowed the therapists to provide nonlinear force to respond to the movement of patients, solving the challenge of traditional software-based rehabilitation system.

Through the cooperative robot based on bilateral teleoperation system, we can get rid of the constraints of distance, environment and so on, and complete the task that the operator cannot complete alone. For example, the teleoperation system studied by Khademian et al. [52] in 2011 allows the trainees and trainers to interact with each other to perform common tasks in a shared virtual environment. In 2013, Shahbazi et al. [53] also proposed a teleoperated dual-user system incorporating Virtual Fixtures (VFs) that allows expert and a trainee conduct a robotic surgery at the same time. The system can adjust the performance and measure the professional level of the trainees.

\section{B. Challenges}

Different from the traditional rigid environment and simple soft environment, the characteristics of complex soft environment are non-elastic and nonlinear, and its environmental parameters and characteristics are time-varying and unstable. In other words, when considering the bilateral teleoperation system involving complex soft environment, the design constraints are different. The trade-off between stability and performance is the main determinant of the control design of bilateral teleoperation system. After checking through the surveyed works in literature, the main difficulties and challenges remaining for further study could be summarized as: First of all, new fidelity evaluation criteria should be considered in addition to conventional impedance-based transparency evaluation in order to accurately describe the interaction with the complex soft environment (e.g., soft tissue under operation). Secondly, it is important to consider and tackle the uncertain factors that affect the stability and transparency of teleoperation, such as physiological tremor of human organs and noise during operation. Especially, when the complex soft environment is active or moving (such as human heart), the bilateral teleoperation system needs to compensate for such motion in order to maintain manipulation stability.

\section{CONCLUSIONS AND PERSPECTIVES}

After surveying and categorizing of recent research works related to bilateral teleoperation with soft environment in literature, it is noticed that the researchers not only employed a variety of control strategies in the bilateral teleoperation system design, but also combined these control strategies with the current advanced machine learning and artificial intelligence techniques to improve the control accuracy and stability. In addition, optimization of the control structure has gradually become one research focus on this topic.

By reviewing the current studies on bilateral teleoperation with soft environments, we believe that the following research directions are of major promise and theoretical/practical importance for the future work: Firstly, delay in communication is still an urgent problem to be solved. Because the soft environment parameters are time-varying, the delay has a great influence on the system performance. Secondly, improving the transparency will still be an important and challenging issue in the design of bilateral teleoperation system taking the soft environment into consideration. Thirdly, the accuracy of current parameter prediction algorithms is not enough to deal with all complex soft environments, more universal and accurate prediction algorithms of environmental parameters are to be further studied. And finally, in terms of practical application, we believe that the bilateral teleoperation of soft environment can extend its application to other fields, such as remote food packaging, unmanned meal delivery, soft object manipulation, remote ultrasonic diagnosis and so on.

\section{REFERENCES}

[1] R. C. Goertz, Master-slave manipulator. Argonne National Lab, 1949.

[2] W. R. Ferrell and T. B. Sheridan, "Supervisory control of remote manipulation," IEEE Spectrum, vol. 4, no. 10, pp. 81-88, 1967.

[3] L. Stark, G. Vossius, and L. R. Young, "Predictive control of eye tracking movements," IRE Transactions on Human Factors in Electronics., no. 2, pp. 52-57, 1962.

[4] R. E. Kalman and J. E. Bertram, "Control system analysis and design via the "second method" of Lyapunov: I-continuous-time systems," 1960.

[5] J. Pratt, C. M. Chew, A. Torres, P. Dilworth, and G. Pratt, "Virtual model control: An intuitive approach for bipedal locomotion," The International Journal of Robotics Research, vol. 20, no. 2, pp. 129-143, 2001.

[6] M. Reed and B. Simon, III: Scattering Theory. Elsevier, 1979.

[7] K. Kasai, Y. Fu, and A. Watanabe, "Passive control systems for seismic damage mitigation," Journal of Structural Engineering, vol. 124, no. 5, pp. 501-512, 1998.

[8] Niemeyer, G., Slotine, and E. J.-J., "Stable adaptive teleoperation," IEEE Journal of Oceanic Engineering, vol. 16, pp. 152-162, 1991.

[9] D. ADAMSKI and R. MILLER, "Unmanned teleoperator spacecraft/UTS/technology," in 6th Annual Meeting and Technical Display, 1969, pp. 1067.

[10] J. Parks and D. Bell, "Industrial robots and machine intelligence," Physics Bulletin , vol. 21, no. 12, pp. 549, 1970.

[11] T. B. Sheridan and W. L. Verplank, "Human and computer control of undersea teleoperators," Massachusetts Institute of Technology Cambridge Man-Machine Systems Lab, 1978.

[12] M. Handlykken and T. Turner, "Control system analysis and synthesis for a six degree-of-freedom universal force-reflecting hand controller," in 19th IEEE Conference on Decision and Control Including the Symposium on Adaptive Processes, 1980, pp. 1197-1205. 
[13] A. K. Bejczy, "Effect of hand-based sensors on manipulator control performance," Mechanism and Machine Theory, vol. 12, no. 5, pp. 547-567, 1977.

[14] J. H. Black, "Factorial study of remote manipulation with transmission of time delay," Massachusetts Institute of Technology, 1971.

[15] R. J. Anderson and M. W. Spong, "Bilateral control of teleoperators with time delay," in IEEE International Conference on Robotics and Automation, vol. 1, pp. 131-138,1988.

[16] A. K. Bejczy, W. S. Kim, and S. C. Venema, "The phantom robot: predictive displays for teleoperation with time delay," in Proceedings, IEEE International Conference on Robotics and Automation, 1990, pp. 546-551.

[17] D. Tesar and W.-K. Kim, "Architectural study of the design and operation of advanced force feedback manual controllers," 1990.

[18] J. PAINES, "An impedance controlled force reflecting hand controller-Design, implementation, and experimentation," in 26th Aerospace Sciences Meeting, 1988, pp. 172.

[19] J. F. Postigo and R. Carelli, "Adaptive Impedance Control for a Bilateral Robot Teleoperation System," IFAC Proceedings Volumes, vol. 33 , no. 27 , pp. $57-62,2000$

[20] J.-Q. Huang, F. L. Lewis, and K. Liu, "A neural net predictive control for telerobots with time delay," Journal of Intelligent and Robotic Systems, vol. 29, pp. 1-25, 2000.

[21] D. A. Lawrence, "Designing teleoperator architectures for transparency," in Proceedings 1992 IEEE International Conference on Robotics and Automation, 1992, pp. 1406-1411.

[22] A. Sherman, M. C. Çavusoglu, and F. Tendick, "Comparison of teleoperator control architectures for palpation task," in Proceedings of the ASME Dynamic Systems and Control Division, Part of the ASME Internatioal Mechanical Engineering Congress and Exposition, 2000, pp. 1261-1268.

[23] U. J. Na, "A new impedance force control of a haptic teleoperation system for improved transparency," Journal of Mechanical Science and Technology, vol. 31, no. 12, pp. 6005-6017, Dec 2017.

[24] J. Bao, H. Wang, and P. X. Liu, "Finite-time Synchronization Control for Bilateral Teleoperation Systems with Asymmetric Time-varying Delay and Input Dead Zone," IEEE/ASME Transactions on Mechatronics, no. 99, pp. 1-1, 2020.

[25] H. Kolbari, S. Sadeghnejad, A. T. Parizi, S. Rashidi, and J. Baltes, "Extended Fuzzy Logic Controller for Uncertain Teleoperation System," 2016 4th Rsi International Conference on Robotics and Mechatronics, pp. 78-83, 2016.

[26] Z. Y. Lu, P. F. Huang, Z. X. Liu, and H. F. Chen, "Fuzzy-Observer-Based Hybrid Force/Position Control Design for a Multiple-Sampling-Rate Bimanual Teleoperation System," IEEE Transactions on Fuzzy Systems, vol. 27, no. 7, pp. 1383-1396, Jul 2019.

[27] A. Hace and K. Jezernik, "Bilateral teleoperation by sliding mode control and reaction force observer," in 2010 IEEE International Symposium on Industrial Electronics, 4-7 July 2010, pp. 1809-1816.

[28] L. R. Salinas et al., "P plus d Plus Sliding Mode Control for Bilateral Teleoperation of a Mobile Robot," International Journal of Control Automation and Systems, vol. 16, no. 4, pp. 1927-1937, Aug 2018.

[29] T. Slama, A. Trevisani, D. Aubry, R. Oboe, and F. Kratz, "Experimental Analysis of an Internet-Based Bilateral Teleoperation System With Motion and Force Scaling Using a Model Predictive Controller," IEEE Transactions on Industrial Electronics, vol. 55,pp. 3290-3299, 2008.

[30] D. Sun, A. Kiselev, Q. F. Liao, T. Stoyanov, and A. Loutfi, "A New Mixed-Reality-Based Teleoperation System for Telepresence and Maneuverability Enhancement," IEEE Transactions Human-Machine System, vol. 50, no. 1, pp. 55-67, Feb 2020.

[31] A. Suzuki and K. Ohnishi, "Frequency-Domain Damping Design for Time-Delayed Bilateral Teleoperation System Based on Modal Space Analysis," IEEE Transactions Industrial Electronics, vol. 60, pp. 177-190, Jan 2013.

[32] G. De Rossi and R. Muradore, "A Bilateral Teleoperation Architecture using Smith Predictor and Adaptive Network Buffering," IFAC Papersonline, vol. 50, pp. 11421-11426, 2017.

[33] M. C. Çavusoglu, A. Sherman, and F. Tendick, "Design of bilateral teleoperation controllers for haptic exploration and telemanipulation of soft environments," IEEE Transactions Robotics Automation, vol. 18, no. 4, pp. 641-647, 2002.
[34] P. Malysz and S. Sirouspour, "Stable non-linear force/position mapping for enhanced telemanipulation of soft environments," Proceedings of the 2007 Ieee International Conference on Robotics and Automation, Vols 1-10, pp. 4307-4312, 2007.

[35] P. Malysz and S. Sirouspour, "Nonlinear and Filtered Force/Position Mappings in Bilateral Teleoperation With Application to Enhanced Stiffness Discrimination," IEEE Transactions on Robotics, vol. 25, no. 5, pp. 1134-1149, 2009.

[36] M. R. Dehghan, S. M. Rezaei, H. A. Talebi, and M. Zareinejad, "Robust High Fidelity Needle Insertion in Soft Tissues Implemented on a Teleoperation System," IFAC Proceedings Volumes, vol. 44, no. 1, pp. 344-349, 2011.

[37] H. Li, K. Tadano, and K. Kawashima, "Model-based passive bilateral teleoperation with time delay," Transactions of the Institute of Measurement and Control, vol. 36, no. 8, pp. 1010-1023, 2014.

[38] L. Xue, Y. Xie, K. M. Zhang, Y. P. Chen, and G. J. Geng, "A Haptic Force Feedback System for Teleoperated Needle Insertion," in 2017 IEEE International Conference on Robotics and Biomimetics pp. 971-976, 2017.

[39] Z. Hu, Y. Y. Wang, G. H. Cui, and D. Zhang, "Enhance Transparency of Force Feedback Interaction Series Mechanism by SMC Strategy," International Journal of Control Automation and Systems, vol. 17, no. 7, pp. 1738-1750, Jul 2019.

[40] M. Minelli, F. Ferraguti, N. Piccinelli, R. Muradore, C. Secchi, "An energy-shared two-layer approach for multi-master-multi-slave bilateral teleoperation systems," International Journal of Control Automation and Systems, pp. 423-429, 2019.

[41] H. Su, Y. Schmirander, Z. Li, X. Zhou, G. Ferrigno, and E. De Momi, "Bilateral teleoperation control of a redundant manipulator with an $\mathrm{rcm}$ kinematic constraint," IEEE International Conference on Robotics and Automation, 2020, pp. 4477-4482.

[42] J. Guo, L. He, and S. Guo, "Study on Force Feedback Control of the Vascular Interventional Surgical Robot based on Fuzzy PID," IEEE International Conference on Mechatronics and Automation, 2020.

[43] A. Talasaz, R. V. Patel, and M. D. Naish, "Haptics-enabled Teleoperation for Robot-assisted Tumor Localization," IEEE International Conference on Robotics and Automation, pp. 5340-5345, 2010.

[44] M. Aoki et al., "Identification Method of Environmental Stiffness using Haptic Forceps for Brain Surgery," IEEE/ASME International Conference on Advanced Intelligent Mechatronics, pp. 207-212, 2018.

[45] C. Liu, P. Moreira, N. Zemiti, and P. Poignet, "3D Force Control for Robotic-Assisted Beating Heart Surgery Based on Viscoelastic Tissue Model," Annual International Conference of the Ieee Engineering in Medicine and Biology Society, pp. 7054-7058, 2011.

[46] X. Q. Bao, S. X. Guo, N. Xiao, Y. X. Li, and L. W. Shi, "Compensatory force measurement and multimodal force feedback for remote-controlled vascular interventional robot," Biomedical Microdevices, vol. 20, no. 3, pp.1-11,2018.

[47] S. X. Guo, Y. Wang, N. Xiao, Y. X. Li, and Y. H. Jiang, "Study on real-time force feedback for a master-slave interventional surgical robotic system," Biomedical Microdevices, vol. 20, no. 2, pp.37, 2018.

[48] J. Shin, Y. M. Zhong, and C. F. Gu, "Master-slave robotic system for needle indentation and insertion," Computer Assisted Surgery, vol. 22, pp. 100-105, 2017.

[49] S. F. Atashzar, I. G. Polushin, and R. V. Patel, "Networked teleoperation with non-passive environment: Application to tele-rehabilitation," in IEEE/RSJ International Conference on Intelligent Robots and Systems, 2012, pp. 5125-5130.

[50] M. Tufail, "Haptic teleoperation with impedance control based on learned inverse dynamics with application in homecare robotics," University of British Columbia, 2015.

[51] S. F. Atashzar, I. G. Polushin, and R. V. Patel, "A Small-Gain Approach for Nonpassive Bilateral Telerobotic Rehabilitation: Stability Analysis and Controller Synthesis," IEEE Transactions on Robotics, vol. 33, no. 1, pp. 49-66, Feb 2017.

[52] B. Khademian, J. Apkarian, and K. Hashtrudi-Zaad, "Assessment of environmental effects on collaborative haptic guidance," Presence: Teleoperators and Virtual Environments, vol. 20, pp. 191-206, 2011.

[53] M. Shahbazi, S. F. Atashzar, and R. V. Patel, "A Dual-User Teleoperated System with Virtual Fixtures for Robotic Surgical Training," IEEE International Conference on Robotics and Automation, pp. 3639-3644, 2013. 